Article

\title{
Circulating and Adipose Tissue Fatty Acid Composition in Black South African Women with Obesity: A Cross-Sectional Study
}

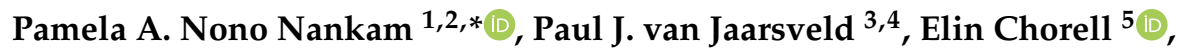 \\ Melony C. Fortuin-de Smidt ${ }^{1}$, Kevin Adams ${ }^{1}$, Matthias Blüher ${ }^{2,6}$, Tommy Olsson ${ }^{5}$ (D, \\ Amy E. Mendham ${ }^{1,3}$ and Julia H. Goedecke 1,3 (D) \\ 1 Division of Exercise Science and Sports Medicine, Department of Human Biology, University of Cape Town, \\ Cape Town 7700, South Africa; mcfortuindesmidt@gmail.com (M.C.F.-d.S.); \\ kevin@academyoplasticsurgery.co.za (K.A.); amymendham21@gmail.com (A.E.M.); \\ julia.goedecke@uct.ac.za (J.H.G.) \\ 2 Department of Endocrinology, Faculty of Medicine, University of Leipzig, 04103 Leipzig, Germany; \\ Matthias.Blueher@medizin.uni-leipzig.de \\ 3 Non-Communicable Diseases Research Unit, South African Medical Research Council, Cape Town 7505, \\ South Africa; Paul.vanJaarsveld@mrc.ac.za \\ 4 Division of Medical Physiology, Faculty of Medicine and Health Sciences, Stellenbosch University, \\ Tygerberg 7505, South Africa \\ 5 Department of Public Health and Clinical Medicine, Umeå University, SE-907 42 Umeå, Sweden; \\ elin.chorell@umu.se (E.C.); tommy.g.olsson@umu.se (T.O.) \\ 6 Helmholtz Institute for Metabolic, Obesity and Vascular Research (HI-MAG) of the Helmholtz Zentrum \\ München at the University of Leipzig and University Hospital Leipzig, 04103 Leipzig, Germany \\ * Correspondence: pamela.nononankam@medizin.uni-leipzig.de; Tel.: +49-341-97-13400
}

Received: 3 April 2020; Accepted: 22 May 2020; Published: 31 May 2020

\begin{abstract}
Background and Aims: During positive energy balance, excess lipid storage in subcutaneous adipose tissue (SAT) is associated with increased lipolysis. Elevated circulating fatty acid (FA) concentrations from both SAT lipolysis and dietary fat intake may result in visceral adipose tissue (VAT) accumulation, impairment of glucose metabolism, altogether increasing obesity-associated metabolic risks. We aimed to test the hypothesis that FA composition of red blood cell total phospholipids (RBC-TPL) and SAT is associated with body fat centralisation (VAT/SAT ratio) and insulin sensitivity $\left(\mathrm{S}_{\mathrm{I}}\right)$ in black South African women with obesity. Methods: Participants' $(n=41)$ body fat composition and distribution, $\mathrm{S}_{\mathrm{I}}$, and RBC-TPL, abdominal and gluteal SAT (gSAT) FA composition (gas-liquid chromatography) were measured. Results: RBC-TPL contained higher proportions of saturated fatty acids (SFAs) than SAT $(p<0.001)$, which were associated with lower $\mathrm{S}_{\mathrm{I}}$ $(p<0.05)$. Mono-unsaturated fatty acids (MUFAs) and stearoyl-CoA desaturase-1 (SCD1)-16 were lower, while poly-unsaturated fatty acids (PUFAs), and delta- 5 and delta- 6 desaturase indices were higher in RBC-TPL than SAT $(p<0.001)$. Interestingly, FA profiles differed between SAT depots with higher SFAs and lower MUFAs, SCD1-16 and SCD1-18 indices in abdominal compared to gluteal SAT $(p<0.01)$. In both SAT depots, higher SFAs and lower PUFAs (n-3 and n-6) correlated with lower VAT/SAT ratio; and lower PUFAs (n-3 and n-6) and higher total MUFA correlated with higher $\mathrm{S}_{\mathrm{I}}$. Conclusion: Our findings confirm the relationships between the FA composition of RBC-TPL and SAT and metabolic risk in black women with obesity, which are dependent on both the FA class, and the tissue type/blood compartment in which they are distributed.
\end{abstract}

Keywords: fatty acids metabolism; desaturase enzyme indices; erythrocytes; subcutaneous adipose tissue; body composition; insulin sensitivity 


\section{Introduction}

Obesity is associated with insulin resistance (IR) and this relationship is dependent on body fat distribution [1-3], which varies between ethnicities [4]. In South Africa (SA), the prevalence of obesity is higher in populations of African ancestry, especially in black women [5] who present with less visceral adipose tissue (VAT) and greater peripheral subcutaneous adipose tissue (SAT) than their white counterparts [4,6]. Paradoxically, despite this 'favorable' body fat phenotype, black African women are more insulin resistant than women of European descent $[4,6]$. The causes of these differences in insulin sensitivity are not yet fully understood and could be derived not only from differences in body fat distribution but also from variations in dietary fat intake $[7,8]$. Indeed, dietary fat intake differs between ethnicities with a lower intake of saturated fatty acid (SFA) and higher n-6 polyunsaturated fatty acid (PUFA) intake in black African women compared to their white counterparts [4,8]. This ethnic-specific dietary intake has been suggested to explain the dissimilarities in fatty acid (FA) metabolism and composition, basal insulin sensitivity and secretion and, consequently, ethnicity-specific variance in insulin sensitivity [9-11].

Long-term dietary fat intake is more accurately evaluated by the measurement of the FA composition of red blood cell total phospholipids (RBC-TPL), compared to plasma or serum FA profiles which only reflect the dietary FA intake of individuals for the last 2-3 weeks [12-15]. Alterations in RBC-TPL FA composition have been reported in insulin-resistant patients and were suggested to be derived either from modifications in dietary FA intake or changes in desaturase enzymes activities such as stearoyl-CoA desaturase-1 (SCD1), delta-5-desaturase (D5D) and delta-6 desaturase (D6D) [16]. Indeed, higher SCD1 and D6D and lower D5D activity have been associated with the impairment of insulin sensitivity in individuals with obesity [17-19]. Moreover, alterations in the composition of cell membrane phospholipids in insulin-sensitive tissues (e.g., AT, liver and muscle) may change membrane fluidity and consequently impair insulin signaling pathways [16].

Adipose tissue (AT) represents the major storage site of excess lipids in the form of triglycerides during positive energy balance, with an increase in adipocyte number and/or size resulting in the expansion of tissue mass [20]. The metabolic effects of AT expansion vary with its location and are dependent on the size of the depot. Indeed, excess lipid storage in SAT may cause the impairment of tissue metabolism and function [21]. Although VAT accumulation is considered to be highly linked to adverse metabolic effects [21], SAT accumulation can also contribute to the development of T2D, especially among black SA women, with several putative mechanisms identified [3]. Among others, increasing SAT basal and/or stimulated lipolysis during obesity elevates circulating free fatty acid (FFA) concentrations, which may interfere with glucose transport/uptake and phosphorylation activity, contributing to impaired peripheral insulin sensitivity [14,22]. Furthermore, these FFAs can be redirected to ectopic regions including VAT, thus, increasing obesity-associated metabolic risks [21]. VAT accumulation may therefore be influenced by SAT lipid metabolism and circulating FA profiles.

Consequently, it has been suggested that the AT depot-specific metabolic effects are partly exerted via differences in lipids and FA metabolism [21,23]. In this regard, the rate of FA synthesis, uptake and release, as well as FA mobilization and endogenous synthesis, differs between SAT depots (mainly abdominal and gluteal SAT) [24-26]. Furthermore, distinctive FA profiles of SAT depots have been reported [27]. However, there is a lack of recent studies comparing the FA composition between SAT depots and investigating their relationship with obesity-associated metabolic risks. We therefore aimed to test the hypothesis that the FA compositions of RBC-TPL and depot-specific SAT are associated with central body fat distribution and insulin sensitivity in black South African women with obesity.

\section{Methods}

\subsection{Study Participants and Ethical Considerations}

Data in this cross-sectional study were baseline data collected as part of a 12-week aerobic-resistance training study, which will be published elsewhere. The detailed design of the study, including 
participant enrollment, inclusion criteria and screening procedures, has been previously published in the protocol paper [28]. Participants were recruited from universities and community groups in Cape Town, SA. They were included in the study if they were women aged between 20-35 years of isiXhosa ancestry (both parents) with stable body weight (i.e., $<5 \%$ change from maximum weight for the last 6 months). Exclusion criteria were: (1) metabolic or inflammatory diseases (e.g., HIV, tuberculosis, active hepatitis, or rheumatoid arthritis), (2) smoking, (3) pregnancy, (4) lactation, (5) use of injectable contraception, which is the common contraception method in this population. Based on these criteria, a total of 41 black SA women with a BMI of $30-40 \mathrm{~kg} / \mathrm{m}^{2}$ could be included in the present study. The study has been approved by the Human Research Ethics Committee of the Faculty of Health Sciences at the University of Cape Town (HREC REF: 827/2016). This study was registered to the Pan-African Clinical Trial Registry (trial registration: PACTR201711002789113) and was performed following the principles of the Declaration of Helsinki (1964, amended last in Fortaleza Brazil, 2013), the International Conference on Harmonization-Good Clinical Practice (ICH-GCP), and the laws of SA. All participants provided verbal and written informed consent before their involvement in any aspect of the study.

In brief, anthropometry, body composition (including VAT and SAT areas) and fat distribution parameters were measured. Insulin sensitivity was measured using a frequently sampled intravenous glucose tolerance test (FSIGT) and SAT samples were collected for the analyses of FA composition. Participants' usual dietary intake was estimated using a self-reported 7-day food frequency questionnaire, and energy and macronutrient intakes were analyzed as previously described [29].

\subsection{Body Composition and Body Fat Distribution}

As previously described [28], basic measures of anthropometry included weight, height, waist circumference (WC) and hip circumference (HC); whole-body composition was measured by dual-energy x-ray absorptiometry (DXA; Discovery-W, software version 12.7.3.7; Hologic, Bedford, MA, USA) according to standard procedures. Regional body fat distribution (gynoid and android fat as a proportion of total fat mass (\%FM)) was characterized as previously described [30,31]. After a standardized meal (Energy: $2553 \mathrm{~kJ}$ ), magnetic resonance imaging (MRI) was used to determine VAT and SAT volumes using a 3 Tesla whole-body human MRI scanner (MAGNETOM Skyra, Siemens Medical Solutions, Erlangen, Germany) as previously described [32].

\subsection{Fasting Blood Samples and Frequently Sampled Intravenous Glucose Tolerance Test}

Participants stayed overnight at the laboratory and were given a standardized evening meal at 8 PM before overnight fasting. The standardized meal was typical of their usual dinner intake, which contained $2456 \mathrm{~kJ}$ energy (E), $21 \mathrm{~g}$ protein (14\% E), $49 \mathrm{~g}$ carbohydrate $(33 \% \mathrm{E})$, and $32 \mathrm{~g}$ fat $(48 \% \mathrm{E})$. At 7 AM, fasting blood samples were collected for the determination of RBC-TPL FA composition. After fasting blood collection, participants underwent an FSIGT. Baseline blood samples were collected (at -5 and $-1 \mathrm{~min})$ before the intravenous administration of glucose $\left(50 \%\right.$ dextrose; $11.4 \mathrm{~g} / \mathrm{m}^{2}$ body surface area) over $60 \mathrm{~s}$. After $20 \mathrm{~min}$, human insulin (0.02 U/kg; NovoRapid, Novo Nordisk) was infused over 5 min (HK400 Hawkmed Syringe Pump, Shenzhen Hawk Medical Instrument Co., Shenzhen, China) and samples were subsequently collected for the determination of plasma glucose and serum insulin concentrations as previously described [28]. Insulin sensitivity $\left(\mathrm{S}_{\mathrm{I}}\right)$ was calculated using Bergman's minimal model of glucose kinetics [33].

\subsection{Adipose Tissue Sample Collection}

Participants underwent fat biopsies (mini-liposuction) for SAT sample collection after 4-6 h of fasting for the subsequent determination of FA composition in abdominal SAT (aSAT; around the umbilicus) and gluteal SAT (gSAT; right upper outer quadrant) as previously described [28]. Approximately $2-3 \mathrm{~cm}^{3}$ of fat tissue was extracted from each site, washed with normal saline until no blood was visible, immediately frozen in liquid nitrogen $\left(\mathrm{N}_{2}\right)$ and stored at $-80^{\circ} \mathrm{C}$ until further analyses. 


\subsection{Determination of RBC-TPL and SAT Fatty Acid Composition}

As previously described in [28], FA composition in RBC-TPL and SAT samples was evaluated using pairwise analyses that ensured samples from a participant were analyzed in the same batch on the same day. Aliquots of saline-washed RBCs $(300 \mu \mathrm{L})$ and SAT $(100 \mathrm{mg})$ were subjected to total lipid extraction with chloroform (amylene stabilised):methanol (2:1; vol:vol; containing 0.01\% butylated hydroxytoluene) by using a modification of the method of Folch et al. [34,35]. Thin-layer chromatography (TLC) was applied to isolate the TPL fraction in the RBC total lipid extract [35]. The TLC-isolated RBC-TPL fractions and a small aliquot of the AT total lipid extracts were trans-methylated using methanol:sulphuric acid (95:5; vol:vol) at $70{ }^{\circ} \mathrm{C}$ for $2 \mathrm{~h}$ to yield fatty acid methyl esters (FAMEs) which, after cooling, were extracted with water and $n$-hexane. The organic layer containing the FAMEs was evaporated, redissolved in a small volume of $n$-hexane and analyzed by gas-liquid chromatography, as described in [35]. The sample FAMEs were identified by comparison of the retention times with those of a standard FAME mixture (27 FAMEs; Nu-Chek Prep Inc., MN, USA). The relative percentage of a FAME was calculated by taking the area under the curve (AUC) of a given FAME as a percentage of the total area count of all the FAMEs identified in the sample (\%, wt:wt). Product-to-precursor FA ratios of the samples were used as a proxy indices to reflect estimated desaturase enzyme activities: 18:3n-6/18:2n-6 (gamma-linolenic acid; GLA/linoleic acid; LA) for D6D and 20:4n-6/20:3n-6 (arachidonic acid; AA/dihomo-gamma-linolenic acid; DGLA) for D5D activity [17,36]. SCD1 activity was estimated by the ratios of 16:1n-7/16:0 (for SCD1-16) and 18:1n-9/18:0 (for SCD1-18) [37]. This method has been well established as an approach to estimate the activity of desaturase enzymes in humans $[17,38,39]$.

\section{Statistical Analyses}

Data were expressed as mean \pm standard deviation (SD) or median interquartile range (25th-75th percentile) depending on the normality of quantitative variables. Normality was tested using the Shapiro-Wilk test and data were log-transformed before analysis if $\mathrm{p}$ values from the normality test were lower than 0.05. The comparison of FA composition between RBC, aSAT and gSAT was performed using a one-way repeated measures ANOVA (using Stata software v.13.1). The relationship between FA profiles in each tissue type and VAT/SAT ratio and $\mathrm{S}_{\mathrm{I}}$ were explored using projection based multivariate analysis. Firstly, the data set was inspected by a principal component analysis (PCA) to detect groupings, trends and outliers. The associations between tissue-specific FA composition and VAT/SAT ratio and $S_{\text {I }}$ were subsequently explored using orthogonal partial least squares of analyses (OPLS). All models were validated based on ANOVA of the cross-validated OPLS scores (CV-ANOVA) for significance testing [40]. All OPLS analyses were performed using SIMCA v.16. FAs were considered significant when fulfilling the statistical significance criteria using post-hoc linear regression on loadings calculated from the validated OPLS models on a 95\% confidence level [41].

\section{Results}

\subsection{Participant Characteristics and Dietary Intake}

The participants involved in this cross-sectional study were young (23 (21-27) years), obese $\left.(33.9 \pm 2.8) \mathrm{kg} / \mathrm{m}^{2}\right)$ women (Table 1). The time course response of the glucose, insulin and c-peptide in response to the FSIGT have been recently published [32]. Self-reported dietary fat intake comprised of $34 \%$ of total energy ( $\%$ E), of which $9.5 \% \mathrm{E}$ was from SFA, $11.4 \% \mathrm{E}$ from MUFA and $7.9 \% \mathrm{E}$ from PUFA (Table 2). 
Table 1. Characteristics of participants.

\begin{tabular}{|c|c|}
\hline Variables & Values $(n=41)$ \\
\hline Age (year) & $23(21-27)$ \\
\hline \multicolumn{2}{|l|}{ Body composition } \\
\hline BMI $\left(\mathrm{kg} / \mathrm{m}^{2}\right)$ & $33.9 \pm 2.8$ \\
\hline Waist circumference $(\mathrm{cm})$ & $103.8 \pm 8.0$ \\
\hline Hip circumference $(\mathrm{cm})$ & $116(114-121)$ \\
\hline WHR & $0.9(0.8-0.9)$ \\
\hline Body FM (\%) & $50.4 \pm 12.0$ \\
\hline Android FM (\%) & $8.1 \pm 1.1$ \\
\hline Gynoid FM (\%) & $18.6 \pm 2.0$ \\
\hline $\operatorname{VAT}\left(\mathrm{cm}^{3}\right)$ & $900 \pm 345$ \\
\hline SAT $\left(\mathrm{cm}^{3}\right)$ & $5454 \pm 1547$ \\
\hline VAT/SAT & $0.2 \pm 0.2$ \\
\hline \multicolumn{2}{|l|}{ Insulin sensitivity } \\
\hline Fasting glucose (mmol/L) & $2777.6(2027.6-4287.5)$ \\
\hline Fasting Insulin $(\mu \mathrm{IU} / \mathrm{mL})$ & $13.1(8 .-16.7)$ \\
\hline $\mathrm{S}_{\mathrm{I}}(\mathrm{mU} / \mathrm{L})^{-1} \min ^{-1}$ & $2.5 \pm 1.4$ \\
\hline
\end{tabular}

Values are expressed as mean \pm SD or median (25th-75th percentile). Body mass index (BMI); fat mass (FM); visceral adipose tissue (VAT); subcutaneous adipose tissue $\mathrm{SAT})$; insulin sensitivity $\left(\mathrm{S}_{\mathrm{I}}\right)$.

Table 2. Dietary energy, macronutrient and fatty acid intake.

\begin{tabular}{cc}
\hline Variables & Median (25th-75th Percentile) \\
\hline Energy (kJ) & $12507(9330-15374)$ \\
Total protein (g) & $96.1(67.5-119.9)$ \\
Protein (\%E) & $13.2(12.2-14.1)$ \\
Total CHO (g) & $372.7(295.1-464.7)$ \\
CHO (\%E) & $53.1(49.0-54.8)$ \\
Total Fat (g) & $113.0(71.5-131.4)$ \\
Fat (\% E) & $33.4(30.1-36.5)$ \\
SFA (g) & $31.4(18.7-42.3)$ \\
SFA (\%E) & $9.5(7.9-11.6)$ \\
MUFA (g) & $36.0(24.1-44.6)$ \\
MUFA (\%E) & $11.4(10.1-12.5)$ \\
PUFA (g) & $25.8(18.0-32.5)$ \\
PUFA (\%E) & $7.9(7.2-9.8)$ \\
\hline
\end{tabular}

Data presented as median (25th and 75th percentiles); $n=41$. Kilojoules (kJ); carbohydrate (CHO); saturated fatty acid (SFA); monounsaturated fatty acid (MUFA); polyunsaturated fatty acid (PUFA).

\subsection{Tissue-Specific Fatty Acid Composition}

The FA composition of RBC-TPL was 44\% SFA, 16\% MUFA and 40\% PUFA, while gSAT comprised of $31 \%$ SFA, $43 \%$ MUFA and $26 \%$ PUFA and aSAT comprised of $32 \%$ SFA, $42 \%$ MUFA and $26 \%$ PUFA (Table 3). Accordingly, total SFA and PUFA were higher, while total MUFA was lower in RBC-TPL compared to SAT depots $(p<0.001)$. When comparing these FA classes between SAT depots, total SFA was higher and total MUFA was lower in aSAT than in gSAT $(p<0.01)$, with no difference in total PUFA content $(p>0.05)$.

When comparing individual FAs in RBC-TPL and SAT depots (Table 3), long-chain SFAs (18:0, 20:0, 22:0 and 24:0) were higher and the medium-chain SFA 14:0 was lower in RBC-TPL than in SAT depots $(p<0.001)$. Notably, 16:0 and 18:0 were higher in aSAT compared to gSAT $(p<0.05)$. Individual MUFAs (16:1n-7, 18:1n-7, 18:1n-9, 20:1n-9) were lower in RBC-TPL compared to SAT depots $(p<0.001)$, and 16:1n-7 and 18:1n-7 were higher in gSAT than in aSAT $(p<0.01)$. In contrast, $n-3$ PUFAs EPA (20:5n-3), DPA n-3 (22:5n-3), DHA (22:6n-3) and total n-3 FAs were higher in RBC-TPL than in SAT depots $(p<0.001)$, with no significant difference between aSAT and gSAT $(p>0.05)$. Likewise, $\mathrm{n}-6$ PUFAs DGLA (20:3n-6), AA (20:4n-6), adrenic acid (22:4n-6), DPA n-6 (22:5n-6) and total n-6 PUFA 
were higher in RBC-TPL than in SAT depots $(p<0.001)$, and only DGLA was higher in gSAT compared to aSAT $(p<0.05)$. Conversely, LA (18:2n-6) and eicosadienoic acid (20:2n.6) contents were lower in RBC-TPL $(p<0.001)$, with no difference between SAT depots $(p>0.05)$. Furthermore, compared to SAT depots, D5D, D6D and SCD1-18 indices were higher $(p<0.001)$, while the SCD1-16 index in RBC-TPL was lower, as mirrored by the product/precursor FA composition $(p<0.001)$. Notably, both SCD1-16 and SCD1-18 indices were higher in gSAT compared to aSAT $(p<0.01)$ with no difference for D5D and D6D indices.

Table 3. Comparison of fatty acid composition (percentage) between red blood cell total phospholipids, gluteal and abdominal subcutaneous adipose tissue depots.

\begin{tabular}{|c|c|c|c|c|}
\hline & RBC-TPL & gSAT & aSAT & $\begin{array}{c}p \\
\text { Values }\end{array}$ \\
\hline \multicolumn{5}{|c|}{ Saturated fatty acids (SFAs) } \\
\hline 14:0 (Myristic acid) & $0.24 \pm 0.06^{\mathrm{a}, \mathrm{b}}$ & $2.77 \pm 0.62$ & $2.89 \pm 0.59$ & $<0.001$ \\
\hline 16:0 (Palmitic acid) & $21.88 \pm 2.06$ & $21.70 \pm 1.12$ & $22.52 \pm 1.11^{\mathrm{c}}$ & 0.024 \\
\hline 18:0 (Stearic acid) & $16.31 \pm 0.78^{a, b}$ & $5.25 \pm 1.53$ & $5.75 \pm 1.49^{c}$ & $<0.001$ \\
\hline 20:0 (Arachidic acid) & $0.35 \pm 0.04^{\mathrm{a}, \mathrm{b}}$ & $0.16 \pm 0.06$ & $0.16 \pm 0.05$ & $<0.001$ \\
\hline 22:0 (Behenic acid) & $1.39 \pm 0.20^{\mathrm{a}, \mathrm{b}}$ & $0.06 \pm 0.03$ & $0.05 \pm 0.01$ & $<0.001$ \\
\hline 24:0 (Lignoceric acid) & $3.99 \pm 0.64^{\mathrm{a}, \mathrm{b}}$ & $0.05 \pm 0.02$ & $0.04 \pm 0.01$ & $<0.001$ \\
\hline Total SFAs & $44.16 \pm 1.68^{a, b}$ & $30.76 \pm 2.88$ & $32.23 \pm 2.46^{c}$ & $<0.001$ \\
\hline \multicolumn{5}{|c|}{ Mono-unsaturated fatty acids (MUFAs) } \\
\hline 16:1n-7 (Palmitoleic acid) & $0.24 \pm 0.07^{\mathrm{a}, \mathrm{b}}$ & $6.61 \pm 2.09^{c}$ & $5.93 \pm 1.68$ & $<0.001$ \\
\hline $18: 1 n-7$ (cis-Vaccenic acid) & $1.11 \pm 0.22^{\mathrm{a}, \mathrm{b}}$ & $3.00 \pm 0.49^{c}$ & $2.79 \pm 0.43$ & $<0.001$ \\
\hline $18: 1 n-9($ Oleic acid $)$ & $10.70 \pm 0.87^{a, b}$ & $32.56 \pm 0.92$ & $32.49 \pm 1.01$ & $<0.001$ \\
\hline 20:1n-9 (Eicosenoic acid) & $0.18 \pm 0.02^{\mathrm{a}, \mathrm{b}}$ & $0.71 \pm 0.10$ & $0.69 \pm 0.11$ & $<0.001$ \\
\hline Total n-7 MUFAs & $1.34 \pm 0.27^{\mathrm{a}, \mathrm{b}}$ & $9.61 \pm 2.50^{c}$ & $8.71 \pm 2.02$ & $<0.001$ \\
\hline Total n-9 MUFAs & $14.23 \pm 0.89^{\mathrm{a}, \mathrm{b}}$ & $33.31 \pm 0.94$ & $33.22 \pm 1.04$ & $<0.001$ \\
\hline Total MUFAs & $15.57 \pm 0.97^{\mathrm{a}, \mathrm{b}}$ & $42.92 \pm 2.64^{c}$ & $41.93 \pm 2.25$ & $<0.001$ \\
\hline \multicolumn{5}{|c|}{ Poly-unsaturated fatty acids (PUFAs) } \\
\hline $20: 5 n-3$ (EPA) & $0.47 \pm 0.23^{\mathrm{a}, \mathrm{b}}$ & $0.09 \pm 0.04$ & $0.08 \pm 0.04$ & $<0.001$ \\
\hline 22:5n-3 (DPA n-3) & $1.96 \pm 0.31^{\mathrm{a}, \mathrm{b}}$ & $0.27 \pm 0.09$ & $0.24 \pm 0.08<$ & $<0.001$ \\
\hline $22: 6 n-3$ (DHA) & $4.45 \pm 0.81^{\mathrm{a}, \mathrm{b}}$ & $0.24 \pm 0.08$ & $0.21 \pm 0.08<$ & $<0.001$ \\
\hline $18: 2 n-6(L A)$ & $11.71 \pm 1.31^{\mathrm{a}, \mathrm{b}}$ & $22.33 \pm 1.64$ & $22.15 \pm 1.55$ & $<0.001$ \\
\hline $\begin{array}{c}20: 2 \mathrm{n}-6 \\
\text { (Eicosadienoic acid) }\end{array}$ & $0.35 \pm 0.04^{\mathrm{a}, \mathrm{b}}$ & $0.50 \pm 0.10$ & $0.48 \pm 0.12$ & $<0.001$ \\
\hline 20:3n-6 (DGLA) & $1.53 \pm 0.22^{\mathrm{a}, \mathrm{b}}$ & $0.43 \pm 0.12^{c}$ & $0.37 \pm 0.12$ & $<0.001$ \\
\hline $20: 4 n-6(A A)$ & $15.74 \pm 1.19^{a, b}$ & $0.73 \pm 0.18$ & $0.67 \pm 0.17$ & $<0.001$ \\
\hline 22:4n-6 (Adrenic acid) & $3.37 \pm 0.59^{a, b}$ & $0.30 \pm 0.09$ & $0.27 \pm 0.09$ & $<0.001$ \\
\hline 22:5n-6 (DPA-n-6) & $0.68 \pm 0.16^{\mathrm{a}, \mathrm{b}}$ & $0.08 \pm 0.04$ & $0.08 \pm 0.03$ & $<0.001$ \\
\hline Total n-3 & $6.90 \pm 1.87^{a, b}$ & $1.76 \pm 0.33$ & $1.66 \pm 0.32$ & $<0.001$ \\
\hline Total n-6 & $33.38 \pm 1.87^{\mathrm{a}, \mathrm{b}}$ & $24.56 \pm 1.73$ & $24.18 \pm 1.64$ & $<0.001$ \\
\hline Total PUFAs & $40.27 \pm 1.77^{a, b}$ & $26.32 \pm 1.95$ & $25.84 \pm 1.80$ & $<0.001$ \\
\hline \multicolumn{5}{|c|}{ Estimated enzyme activities/indices } \\
\hline D5D & $10.57 \pm 1.95^{\mathrm{a}, \mathrm{b}}$ & $1.79 \pm 0.43$ & $1.90 \pm 0.37$ & $<0.001$ \\
\hline D6D & $0.13 \pm 0.02^{\mathrm{a}, \mathrm{b}}$ & $0.019 \pm 0.01$ & $0.016 \pm 0.01$ & $<0.001$ \\
\hline SCD1-16 & $0.01 \pm 0.00^{\mathrm{a}, \mathrm{b}}$ & $0.31 \pm 0.11^{c}$ & $0.27 \pm 0.08$ & $<0.001$ \\
\hline SCD1-18 & $0.68 \pm 0.01^{\mathrm{a}, \mathrm{b}}$ & $0.65 \pm 0.30^{c}$ & $0.53 \pm 0.21$ & $<0.001$ \\
\hline
\end{tabular}

Fatty acids (FA) presented as relative percentages of total FAs (\%, wt:wt) and mean $\pm \mathrm{SD}(n=41) . p$-Values represent the differences in FA composition between the sites using one-way repeated measures ANOVA as follows: ${ }^{a}$ : RBC

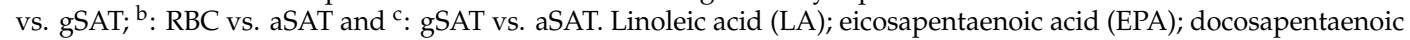
acid (DPA); docosahexaenoic acid (DHA); dihomo-gamma-linolenic acid (DGLA); arachidonic acid (AA); saturated fatty acid (SFA); monounsaturated fatty acid (MUFA); polyunsaturated fatty acid (PUFA); delta-5-desaturase index (D5D) (20:4n-6/20:3n-6); delta-6-desaturase index (D6D) (18:3n-6/18:2n-6); stearoyl-CoA desaturase-1 (SCD1-16: 16:1n-7/16:0; SCD1-18: 18:1n-9/18:0). 


\subsection{Relationship between Fatty Acid Profiles and the VAT/SAT Ratio}

Given the differences in FA composition between RBC-TPL and SAT depots, we then explored the relationships between site-specific FA profiles and the VAT/SAT ratio (Figure 1). In the RBC-TPL, MUFAs (16:1n-7 and 18:1n-7, n-7 MUFA) and the SCD1-16 index were positively associated, while DGLA was negatively associated with the VAT/SAT ratio. The pattern of association between VAT/SAT ratio and SFA and PUFA were similar in both SAT depots. Indeed, lower SFAs (12:0, 14:0, 18:0, 20:0 and total SFA) and higher PUFAs (ALA, AA, DPA n-3, total PUFA, total n-6, total n-3) and the SCD1-18 index were associated with higher VAT/SAT ratio in both SAT depots. However, while vaccenic acid (18:1n-7) was positively associated with VAT/SAT ratio in gSAT, oleic acid (18:1n-9) and total n-9 MUFA in aSAT were inversely correlated with the VAT/SAT ratio.

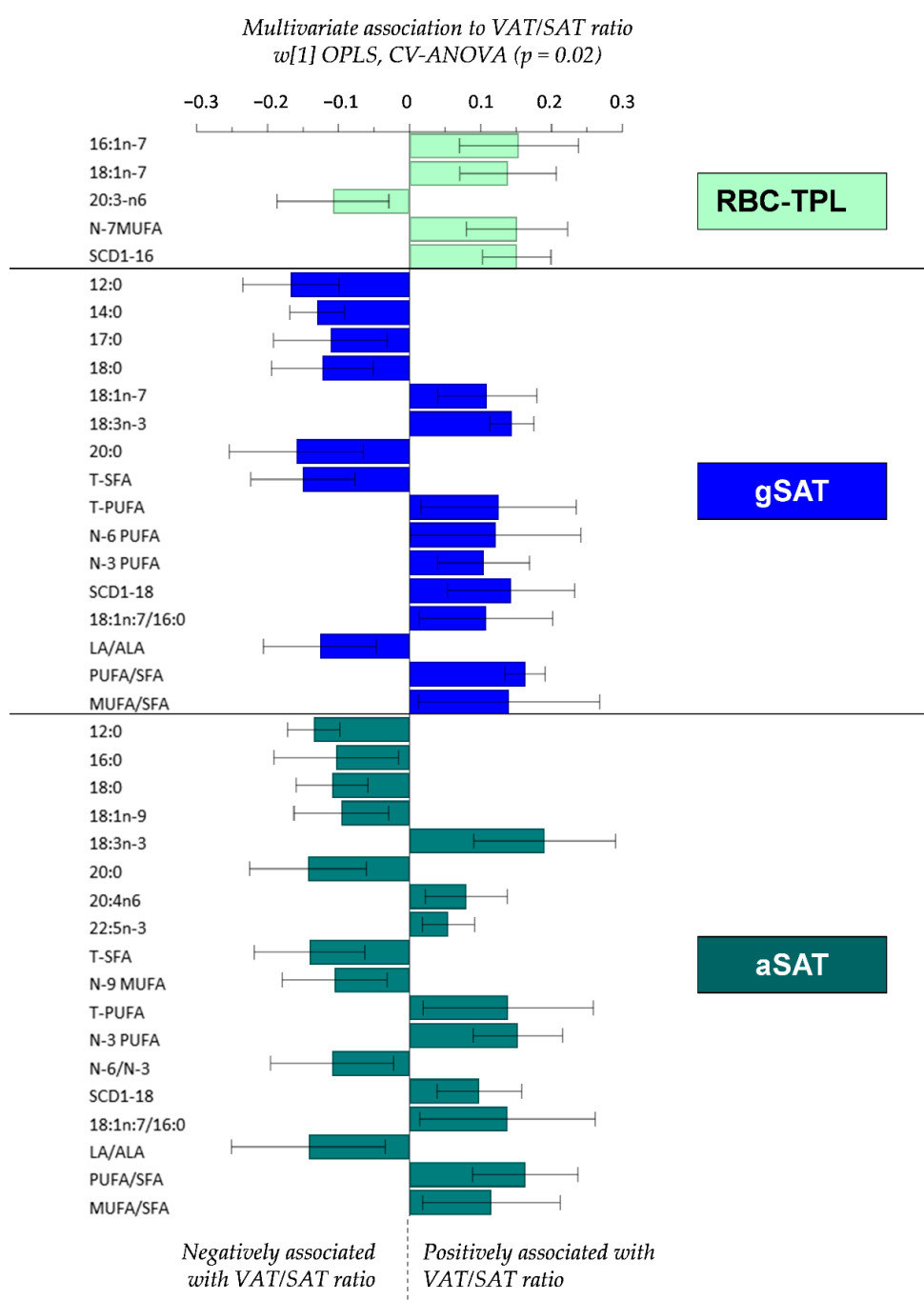

Figure 1. Multivariate OPLS associations between tissue-specific fatty acid composition and the VAT/SAT ratio. Only the individual and grouped FA and FA ratios in each tissue type that were significantly (95\% confidence level) associated with the VAT/SAT ratio are shown in the figure. All fatty acids shown in Table 3 were included in the OPLS model. Total saturated fatty acid (T-SFA); total monounsaturated fatty acid (T-MUFA); total polyunsaturated fatty acid (T-PUFA); linoleic acid (LA); alpha-linolenic acid (ALA); stearoyl-CoA desaturase-1 index (SCD1-16: 16:1n-7/16:0; SCD1-18: 18:1n-9/18:0); 18:1n-7/16:0: vaccenic acid (elongation product of 16:1n7)/palmitic acid; gluteal subcutaneous adipose tissue (gSAT); abdominal subcutaneous adipose tissue (aSAT). 


\subsection{Relationship between Fatty Acid Profiles and $S_{I}$}

The relationships between FA profiles (RBC-TPL and SAT depots) and $\mathrm{S}_{\mathrm{I}}$ are presented in Figure 2. Higher RBC-TPL total SFA and 20:2n-6, and lower 22:4n-6 were associated with lower S. In gSAT, PUFAs (LA, DGLA, DPA n-3, total n-6, total PUFA) and SCD1-18 and D6D indices were inversely correlated with $S_{I}$, while MUFAs (16:1n-7, total n-7 and total MUFA) were positively associated with $\mathrm{S}_{\mathrm{I}}$. Likewise, total MUFA in aSAT were positively associated with $\mathrm{S}_{\mathrm{I}}$, while individual PUFA (LA, eicosadienoic acid, DGLA, adrenic acid, DPA n-3, DHA, total n-6, total PUFA) and the D6D index were negatively associated with $\mathrm{S}_{\mathrm{I}}$.

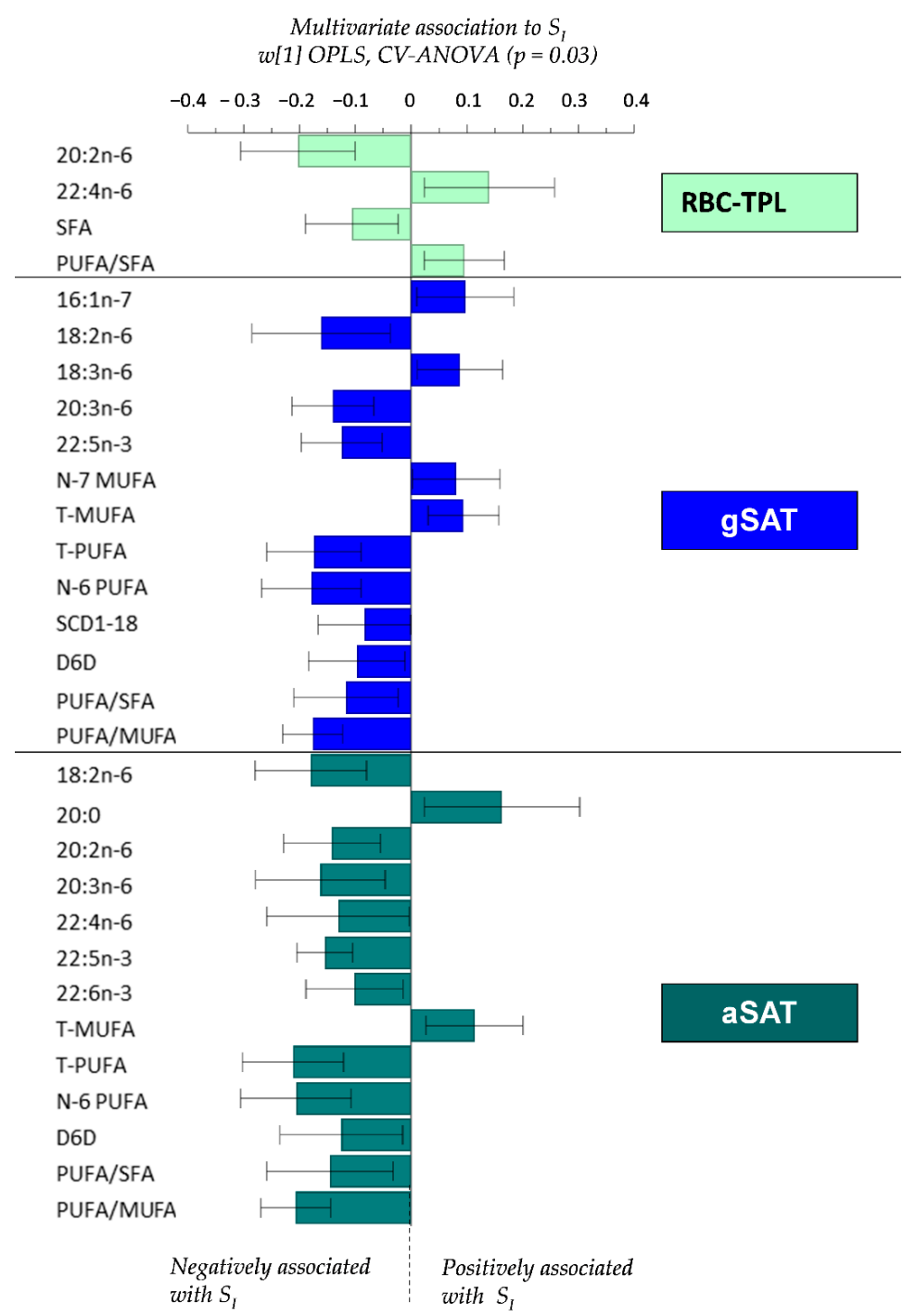

Figure 2. Multivariate OPLS associations between tissue-specific fatty acid composition and $\mathrm{S}_{\mathrm{I}}$. Only the individual and grouped FA and FA ratios in each tissue type that were significantly $(95 \%$ confidence level) associated with $S_{I}$ are presented in the figure. All fatty acids shown in Table 3 were included in the OPLS model. Total saturated fatty acid (T-SFA); total monounsaturated fatty acid (T-MUFA); total polyunsaturated fatty acid (T-PUFA); linoleic acid (LA); alpha-linolenic acid (ALA); delta-6-desaturase index (D6D) (18:3n-6/18:2n-6); stearoyl-CoA desaturase-1 index (SCD1-16: 16:1n-7/16:0; SCD1-18: 18:1n-9/18:0); gluteal subcutaneous adipose tissue (gSAT); abdominal subcutaneous adipose tissue (aSAT). 


\section{Discussion}

The key finding of this study is that the FA composition of circulating phospholipids (RBC-TPL) and SAT are distinctly associated with the centralization of body fat and $\mathrm{S}_{\mathrm{I}}$ in black South African women with obesity. Notably, the FA composition of RBC-TPL was different from FA profiles in SAT. RBC-TPL contained higher proportions of SFAs and PUFAs and lower MUFAs than SAT. The higher content of SFA in RBC-TPL was not associated with the VAT/SAT ratio, but rather with lower $\mathrm{S}_{\mathrm{I}}$. Furthermore, the D5D, D6D and SCD1-18 indices were higher, and the SCD1-16 index was lower in RBC-TPL compared to SAT. Interestingly, the FA composition of SAT depots differed, with higher SFAs (16:0, 18:0 and total SFA) and lower MUFAs (16:1n-7, 18:1n-7 and total MUFA), as well as lower SCD1-16 and SCD1-18 index in aSAT compared to gSAT. Despite these differences, the associations with VAT/SAT ratio and $S_{I}$ did not differ. Indeed, in both SAT depots, lower SFAs and higher PUFAs (n-3 and n-6) correlated with a higher VAT/SAT ratio. Further, lower PUFAs (n-3 and n-6) and higher total MUFA were associated with higher $\mathrm{S}_{\mathrm{I}}$. These findings confirm the hypothesis that the association of FAs with metabolic status is dependent on the FA class and the blood compartment/fraction where they occur or tissue type in which they are stored. The multivariate models were most significant for the FA composition of SAT depots, highlighting a stronger influence of SAT FA metabolism over dietary FA intake (reflected by RBC-TPL) on obesity-associated metabolic risk in these women. These findings support the contribution of excess SAT accumulation in the incidence of metabolic disorders [14,22,42].

The finding of high proportions of SFA and PUFA in RBC-TPL may indicate a high intake of these FA types in black SA women, which is commensurate with previous research in this population [43]. However, this was not supported by the self-reported dietary intake of the participants in this study, which reported lower SFA $(9.5 \% \mathrm{E})$ and PUFA $(8 \% \mathrm{E})$ intake than MUFA intake $(11 \% \mathrm{E})$. It is important to note that under-reporting of dietary intake is well recognized, and may significantly influence investigations of nutrient patterns and association with diseases [13]. Nevertheless, SFAs such as 16:0 can be synthesized endogenously from acetyl-CoA during de novo lipogenesis, and subsequently elongated (to 18:0) and desaturated to generate MUFAs (such as 16:1 and 18:1) [14,16,38]. This pathway is elevated in individuals with obesity, and under high-carbohydrate loads and excess energy intake [14], which is characteristic of the participants in the present study. Excess consumption of carbohydrates and high glycemic load increase lipogenesis with the synthesis of SFA, especially of 16:0 and 18:0 appearing in the circulation $[44,45]$. Accordingly, these were the most abundant SFAs in RBC-TPL in the present study (16:0 (22\%) and 18:0 (16\%)). However, RBC cannot undergo de novo phospholipid synthesis or FA desaturation; these cells renew their membrane FA composition by direct exchange with plasma FFA and phospholipids pools [12]. Therefore, high content of SFA and PUFA in RBC-TPL could be derived from plasma FFAs, highly influenced by both dietary intake and endogenous synthesis in other tissues such as AT and liver. Further studies are required to elucidate the different origins of these FA classes and proportions in RBC membranes.

In contrast to higher content of SFA and PUFA in RBC-TPL, we further report lower MUFA contents in this fraction compared to SAT depots. Although, to our knowledge, no previous studies have compared the FA composition between RBC-TPL and SAT, previous data showed high SFA and PUFA and lower MUFA levels in RBC-TPL of individuals with obesity compared to lean individuals [46,47]. Moreover, we found higher indices of desaturation rates (estimated SCD1-16 activity) in SAT depots than those reflected in RBC-TPL product/precursor FA composition. SCD1-16 is involved in the endogenous synthesis of MUFA from SFA and is mainly active in AT, where it plays a central role for de novo synthesis and storage of excess energy as triglycerides [17]. Accordingly, oleic acid (18:1n-9) has been reported as the major species in adipose triglycerides [12,44,48-50]. Similarly, oleic acid was the most abundant FA in SAT depots (32.5\%) in our study. Moreover, MUFA content in aSAT was lower (and higher SFA content) than that of gSAT in these women, which is parallel to what has been previously found in European populations [12,51]. Differences in FA metabolism between SAT depots (including synthesis, uptake, release, deposition rate, mobilisation and endogenous synthesis) have been reported [24-26]. These differences were attributed to their distinct FA profiles [27] and suggested 
to be dependent on the energy balance [51]. The higher saturation of aSAT has also been linked to the physical properties of this depot (semi-solid) concerning its anatomical role in the protection of abdominal organs [51]. Conversely, the lower content of SFA in gSAT in the present study was complemented by the higher SCD1 activity and a higher percentage of MUFA $(16: 1 n-7,18: 1 n-7$ and total MUFA). This suggests a higher desaturation rate and therefore, a preferential accumulation of MUFA in gSAT. However, PUFA content was similar between aSAT and gSAT, which is supported by previous studies [12,44,51-53]. Long-chain PUFAs are synthesized from essential FAs (18:2n-6 and 18:3n-3) that can only be obtained from food [44,51]. These data support the notion that PUFA content in SAT depots is dependent on dietary intake, with 18:2n-6 representing the major dietary PUFA in SAT. This comparable metabolism of PUFA between aSAT and gSAT was further supported by the lack of difference in estimated desaturase activities observed in the present study.

In addition to the distinct FA profiles of RBC-TPL and SAT depots shown in the present study, it has been previously reported that the metabolic effects of individual FAs are dependent on their respective class [54,55]. For instance, while high MUFA and PUFA contents have been proposed to increase cell membrane fluidity, high contents of SFAs and low MUFAs increase membrane rigidity resulting in a reduced number of insulin receptors and binding affinity in insulin-sensitive cells [14,16]. This evidence might explain, at least in part, the different associations of FA classes with metabolic risks [16]. However, despite differential SFA and MUFA composition in SAT depots in the present study, there were no differences in the association of these FA classes with the centralization of body fat and $S_{I}$. Indeed, lower individual and total SFAs in both SAT depots correlated with a higher VAT/SAT ratio. In contrast, higher total SFA in RBC-TPL, but not in SAT depots, was associated with lower $S_{I}$. These findings are similar to a previous study in black SA individuals showing a positive association between SFAs in plasma phospholipids and measures of adiposity and metabolic syndrome [50]. High intake of dietary SFA may significantly increase IR via alterations of desaturases activity (mainly D5D and D9D) in cell membrane phospholipids and inhibition of insulin-stimulated Akt activation followed by the reduction in glucose uptake $[16,56]$.

In contrast to circulating SFAs, the implication of PUFAs in the development of obesity-associated metabolic disorders is controversial. For instance, Gunes et al. showed an association between membrane enrichment in n-3 PUFA and improvement of $S_{\text {I }}$ in overweight adolescents [14]; and plasma total n-3 PUFA was negatively associated with markers of obesity and metabolic syndrome [57,58]. Moreover, rats fed with diets rich in n-6 PUFA exhibited an increased insulin-stimulated glucose uptake in adipocytes [59]. In contrast, other studies reported positive associations between n-6 PUFA intake, obesity and metabolic syndrome $[60,61]$, as well as inverse associations between RBC total n-6 PUFA and metabolic syndrome $[62,63]$. These discrepancies in the association between circulating PUFAs and metabolic status were also observed in the present study where RBC-TPL adrenic acid (22:4n-6) was positively and eicosadienoic acid (20:2n-6) was negatively correlated with $\mathrm{S}_{\mathrm{I}}$. Further, both n-6 and n-3 PUFAs in SAT depots were negatively associated with $S_{I}$ and positively correlated with the centralization of body fat (VAT/SAT ratio). Our findings are in accordance with a study in a French population showing positive associations between n-6 PUFA content of SAT (abdominal) and central fat distribution [44]. Moreover, studies in Swedish cohorts reported a positive association between individual n-3 PUFA (EPA and DHA) intake and central body fat [64] and positive correlations between aSAT n-6 PUFA (20:3n-6 and 20:4n-6) content and IR [65]. Further, Iggman et al. showed strong positive correlations between individual SFAs content in gSAT (12:0, 14:0 and 18:0) and LA with insulin sensitivity, while DHA negatively correlated with insulin sensitivity [66]. Conversely, high SFA intake was related to a higher deposition of VAT and total body fat, whereas increased dietary PUFA (mainly LA) correlated with reduced central fat accumulation [64]. These data highlight the complexity in the relationship between FA classes (especially PUFAs) and obesity-associated metabolic diseases. The role of circulating and SAT FAs species in central fat accumulation and the impairment of insulin sensitivity in different populations warrants further investigations. 
The limitations of our study include the estimation of desaturase enzyme activities based on product to precursor FA ratios (indices), which may indicate a higher proportion of product than precursor and not necessarily the direct activity of these main desaturases. Moreover, the lack of detailed information on the dietary FA intake may also represent a limitation. However, the robust measure of the FA composition of RBC-TPL and two main depots of SAT represent strengths of this study. This method is regarded as more objective and accurate than estimations from dietary reporting in the investigation of the effects of these lipid molecules in the development of metabolic diseases [12,13]. This study included SA women with obesity and prospective studies in a more representative population (including men, and normal-weight individuals) with a control group are required to gain a better understanding of the associations between RBC-TPL and SAT FA composition and central fat accumulation and development of IR.

In conclusion, we show that the circulating (RBC-TPL) FA profile is distinctly different from the FA composition of SAT, with higher SFAs and PUFAs and lower MUFAs in RBC-TPL compared to the SAT depots. Notably, differences were also observed within SAT depots with higher SFAs and lower MUFAs in aSAT compared to gSAT, which were not reflected in the relationship with the VAT/SAT ratio and $\mathrm{S}_{\mathrm{I}}$. SAT depots, rather than RBC-TPL, were the major contributors to the variance in central fat accumulation and insulin sensitivity. Our findings confirm the hypothesis that the FA composition of RBC-TPL and SAT is associated with metabolic risk in black SA women with obesity; and these associations are not only dependent on the FA class, but also on the tissue type and blood compartment or fraction in which they are distributed. Intervention studies are required to elucidate the causal relationship between FA composition and the metabolic profile, as well as the specific roles of individual FAs and total FA groups in mediating obesity-associated impairment of insulin sensitivity.

Author Contributions: Conceptualization, P.A.N.N., A.E.M. and J.H.G.; data curation, P.A.N.N., P.J.v.J. and E.C.; formal analysis, P.A.N.N., E.C. and J.H.G.; funding acquisition, T.O. and J.H.G.; investigation, P.A.N.N., M.C.F.-d.S., M.B., T.O., A.E.M. and J.H.G.; methodology, P.J.v.J., E.C., M.C.F.-d.S., K.A., A.E.M. and J.H.G.; project administration, A.E.M. and J.H.G.; resources, K.A. and M.B.; software, E.C.; supervision, J.H.G.; validation, P.A.N.N., P.J.v.J., E.C., M.C.F.-d.S., K.A., M.B., T.O., A.E.M. and J.H.G.; visualization, P.A.N.N., P.J.v.J., M.B., T.O., A.E.M. and J.H.G.; writing-original draft, P.A.N.N.; writing—review \& editing, P.J.v.J., E.C., M.C.F.-d.S., K.A., M.B., T.O., A.E.M. and J.H.G. All authors have read and agreed to the published version of the manuscript.

Acknowledgments: This study was funded by the National Research Foundation of South Africa (NRF), Competitive Programme for Rated Researchers (Grant no: 93577). We would like to thank Johanna van Wyk of the Non-Communicable Diseases Research Unit of the South African Medical Research Council (SAMRC) for her helpful laboratory assistance with fatty acid analysis and the SAMRC for supplementary funding. We also thank the participants of this study, the field workers, technicians and laboratory staff of the Division of Exercise Science and Sports Medicine at the University of Cape Town, specifically Nandipha Sinyanya, Ntombekhaya Zoneleni, Keitumetse Smouse and Hendriena Victor, who assisted with the recruitment, intervention and sample analysis.

Conflicts of Interest: The authors declare no conflict of interest.

\section{References}

1. Shay, C.M.; Carnethon, M.R.; Church, T.R.; Hankinson, A.L.; Chan, C.; Jacobs, D.R., Jr.; Lewis, C.E.; Schreiner, P.J.; Sternfeld, B.; Sidney, S. Lower extremity fat mass is associated with insulin resistance in overweight and obese individuals: The CARDIA study. Obesity 2011, 19, 2248-2253. [CrossRef] [PubMed]

2. Preis, S.R.; Massaro, J.M.; Robins, S.J.; Hoffmann, U.; Vasan, R.S.; Irlbeck, T.; Meigs, J.B.; Sutherland, P.; D'Agostino, R.B., Sr.; O'donnell, C.J.; et al. Abdominal subcutaneous and visceral adipose tissue and insulin resistance in the Framingham heart study. Obesity 2010, 18, 2191-2198. [CrossRef] [PubMed]

3. Goedecke, J.H.; Levitt, N.S.; Evans, J.; Ellman, N.; Hume, D.J.; Kotze, L.; Tootla, M.; Victor, H.; Keswell, D. The role of adipose tissue in insulin resistance in women of African ancestry. J. Obes. 2013, 2013, 952916. [CrossRef] [PubMed]

4. Goedecke, J.H.; Levitt, N.S.; Utzschneider, K.M.; Faulenbach, M.V.; Dave, J.A.; West, S.; Victor, H.; Evans, J.; Olsson, T.; Walker, B.R.; et al. Differential effects of abdominal adipose tissue distribution on insulin sensitivity in black and white South African women. Obesity 2009, 17, 1506-1512. [CrossRef] 
5. Ng, M.; Fleming, T.; Robinson, M.; Thomson, B.; Graetz, N. Global, regional, and national prevalence of overweight and obesity in children and adults during 1980-2013: A systematic analysis for the Global Burden of Disease Study 2013. Lancet 2014, 384, 766-781. [CrossRef]

6. Keswell, D.; Tootla, M.; Goedecke, J.H. Associations between body fat distribution, insulin resistance and dyslipidaemia in black and white South African women. Cardiovasc. J. Afr. 2016, 27, 177-183. [CrossRef]

7. Joubert, J.; Norman, R.; Bradshaw, D.; Goedecke, J.H.; Steyn, N.P.; Puoane, T. Estimating the burden of disease attributable to excess body weight in South Africa in 2000. S. Afr. Med. J. 2007, 97, 683-690.

8. Joffe, Y.T.; van der Merwe, L.; Evans, J.; Collins, M.; Lambert, E.V.; September, A.V.; Goedecke, J.H. Interleukin-6 Gene Polymorphisms, Dietary Fat Intake, Obesity and Serum Lipid Concentrations in Black and White South African Women. Nutrients 2014, 6, 2436-2465. [CrossRef]

9. Goff, L.M.; Griffin, B.A.; Lovegrove, J.A.; Sanders, T.A.; Jebb, S.A.; Bluck, L.J.; Frost, G.S. Ethnic differences in beta-cell function, dietary intake and expression of the metabolic syndrome among UK adults of South Asian, black African-Caribbean and white-European origin at high risk of metabolic syndrome. Diabetes Vasc. Dis. Res. 2013, 10, 315-323. [CrossRef]

10. Mathias, R.A.; Sergeant, S.; Ruczinski, I.; Torgerson, D.G.; Hugenschmidt, C.E. The impact of FADS genetic variants on $\omega 6$ polyunsaturated fatty acid metabolism in African Americans. BMC Genet. 2011, 12, 50. [CrossRef]

11. Sergeant, S.; Hugenschmidt, C.E.; Rudock, M.E.; Ziegler, J.T.; Ivester, P.; Ainsworth, H.C.; Vaidya, D.; Case, D.; Langefeld, C.D.; Freedman, B.I.; et al. Differences in arachidonic acid levels and fatty acid desaturase (FADS) gene variants in African Americans and European Americans with diabetes or the metabolic syndrome. $\mathrm{Br}$. J. Nutr. 2011, 107, 547-555. [CrossRef] [PubMed]

12. Hodson, L.; Skeaff, C.M.; Fielding, B.A. Fatty acid composition of adipose tissue and blood in humans and its use as a biomarker of dietary intake. Prog. Lipid Res. 2008, 47, 348-380. [CrossRef] [PubMed]

13. Markussen, M.S.; Veierod, M.B.; Ursin, G.; Andersen, L.F. The effect of under-reporting of energy intake on dietary patterns and on the associations between dietary patterns and self-reported chronic disease in women aged 50-69 years. Br. J. Nutr. 2016, 116, 547-558. [CrossRef] [PubMed]

14. Gunes, O.; Tascilar, E.; Sertoglu, E.; Tas, A.; Serdar, M.A.; Kaya, G.; Kayadibi, H.; Ozcan, O. Associations between erythrocyte membrane fatty acid compositions and insulin resistance in obese adolescents. Chem. Phys. Lipids 2014, 184, 69-75. [CrossRef] [PubMed]

15. Rise, P.; Eligini, S.; Ghezzi, S.; Colli, S.; Galli, C. Fatty acid composition of plasma, blood cells and whole blood: Relevance for the assessment of the fatty acid status in humans. Prostaglandins Leukot Essent Fatty Acids 2007, 76, 363-369. [CrossRef] [PubMed]

16. Perona, J.S. Membrane lipid alterations in the metabolic syndrome and the role of dietary oils. Biochim. Biophys. Acta 2017, 1859, 1690-1703. [CrossRef]

17. Warensjo, E.; Rosell, M.; Hellenius, M.L.; Vessby, B.; de Faire, U.; Riserus, U. Associations between estimated fatty acid desaturase activities in serum lipids and adipose tissue in humans: Links to obesity and insulin resistance. Lipids Health Dis. 2009, 8, 37. [CrossRef]

18. Mahendran, Y.; Agren, J.; Uusitupa, M.; Cederberg, H.; Vangipurapu, J.; Stancakova, A.; Schwab, U.; Kuusisto, J.; Laakso, M. Association of erythrocyte membrane fatty acids with changes in glycemia and risk of type 2 diabetes. Am. J. Clin. Nutr. 2014, 99, 79-85. [CrossRef]

19. Sjogren, P.; Sierra-Johnson, J.; Gertow, K.; Rosell, M.; Vessby, B.; de Faire, U.; Hamsten, A.; Hellenius, M.L.; Fisher, R.M. Fatty acid desaturases in human adipose tissue: Relationships between gene expression, desaturation indexes and insulin resistance. Diabetologia 2008, 51, 328-335. [CrossRef]

20. Roberts, R.; Hodson, L.; Dennis, A.L.; Neville, M.J.; Humphreys, S.M.; Harnden, K.E.; Micklem, K.J.; Frayn, K.N. Markers of de novo lipogenesis in adipose tissue: Associations with small adipocytes and insulin sensitivity in humans. Diabetologia 2009, 52, 882-890. [CrossRef]

21. Blüher, M. Adipose Tissue Dysfunction Contributes to Obesity Related Metabolic Diseases. Best Pract. Res. Clin. Endocrinol. Metab. 2013, 27, 163-177. [CrossRef] [PubMed]

22. Roden, M.; Price, T.B.; Perseghin, G.; Petersen, K.F.; Rothman, D.L.; Cline, G.W.; Shulman, G.I. Mechanism of free fatty acid induced insulin resistance in humans. J. Clin. Investig. 1996, 97, 2859-2865. [CrossRef] [PubMed]

23. Scherer, P.E. Adipose tissue: From lipid storage compartment to endocrine organ. Diabetes 2006, 55, 1537-1545. [CrossRef] [PubMed] 
24. Karastergiou, K.; Fried, S.K.; Xie, H.; Lee, M.J.; Divoux, A.; Rosencrantz, M.A.; Chang, R.J.; Smith, S.R. Distinct developmental signatures of human abdominal and gluteal subcutaneous adipose tissue depots. J. Clin. Endocrinol. Metab. 2013, 98, 362-371. [CrossRef]

25. Karpe, F.; Pinnick, K.E. Biology of upper-body and lower-body adipose tissue-link to whole-body phenotypes. Nat. Rev. Endocrinol. 2015, 11, 90-100. [CrossRef]

26. Karastergiou, K.; Smith, S.R.; Greenberg, A.S.; Fried, S.K. Sex differences in human adipose tissues-The biology of pear shape. Biol. Sex Differ. 2012, 3, 13. [CrossRef]

27. Nikolaidis, M.G.; Mougios, V. Effects of Exercise on the Fatty-Acid Composition of Blood and Tissue Lipids. Sports Med. Open 2004, 34, 1051-1076. [CrossRef]

28. Goedecke, J.H.; Mendham, A.E.; Clamp, L.; Nono Nankam, P.A.; Fortuin-de Smidt, M.C.; Phiri, L.; Micklesfield, L.K.; Keswell, D.; Woudberg, N.J.; Lecour, S.; et al. An Exercise Intervention to Unravel the Mechanisms Underlying Insulin Resistance in a Cohort of Black South African Women: Protocol for a Randomized Controlled Trial and Baseline Characteristics of Participants. JMIR Res. Protoc. 2018, 7, e75. [CrossRef]

29. Clamp, L.D.; Mendham, A.E.; Kroff, J.; Goedecke, J.H. Higher baseline fat oxidation promotes gynoid fat mobilization in response to a 12 week exercise intervention in sedentary, obese black South African women. Appl. Physiol. Nutr. Metab. 2019. [CrossRef]

30. Goedecke, J.H.; Micklesfield, L.K.; Levitt, N.S.; Lambert, E.V.; West, S.; Maartens, G.; Dave, J.A. Effect of different antiretroviral drug regimens on body fat distribution of HIV-infected South African women. AIDS Res. Hum. Retrovir. 2013, 29, 557-563. [CrossRef]

31. Micklesfield, L.K.; Goedecke, J.H.; Punyanitya, M.; Wilson, K.E.; Kelly, T.L. Dual-energy X-ray performs as well as clinical computed tomography for the measurement of visceral fat. Obesity 2012, 20, 1109-1114. [CrossRef] [PubMed]

32. Fortuin-de Smidt, M.C.; Mendham, A.E.; Hauksson, J.; Hakim, O.; Stefanovski, D.; Clamp, L.; Phiri, L.; Swart, J.; Goff, L.M.; Micklesfield, L.K.; et al. Effect of exercise training on insulin sensitivity, hyperinsulinemia and ectopic fat in black South African women: A randomized controlled trial. Eur. J. Endocrinol. 2020. [CrossRef]

33. Bergman, R.; Ider, Y.; Bowden, C.; Cobelli, C. Quantitative estimation of insulin sensitivity. Am. J. Physiol. 1979, 236, E667-E677. [CrossRef]

34. Folch, J.; Lees, M.; Sloane Stanley, G.H. A simple method for the isolation and purification of total lipids from animal tissues. J. Biol. Chem. 1957, 226, 497-509. [PubMed]

35. Chimhashu, T.; Malan, L.; Baumgartner, J.; van Jaarsveld, P.J.; Galetti, V.; Moretti, D.; Smuts, C.M.; Zimmermann, M.B. Sensitivity of fatty acid desaturation and elongation to plasma zinc concentration: A randomised controlled trial in Beninese children. Br. J. Nutr. 2018, 119, 610-619. [CrossRef]

36. Fan, Y.Y.; Chapkin, R.S. Importance of dietary $\gamma$-linolenic acid in human health and nutrition. J. Nutr. 1998, 128, 1411-1414. [CrossRef] [PubMed]

37. Jump, D.B. Fatty acid regulation of hepatic lipid metabolism. Curr. Opin. Clin. Nutr. Metab. Care 2011, 14, 115-120. [CrossRef]

38. Kroger, J.; Zietemann, V.; Enzenbach, C.; Weikert, C.; Jansen, E.H.J.M.; Döring, F.; Joost, H.; Boeing, H.; Schulze, M.B. Erythrocyte membrane phospholipid fatty acids, desaturase activity, and dietary fatty acids in relation to risk of type 2 diabetes in the European prospective investigation into cancer and nutrition (EPIC)-potsdam study. Am. J. Clin. Nutr. 2011, 93, 127-142. [CrossRef]

39. Martinelli, N.G.D.; Malerba, G.; Guarini, P.; Illig, T.; Trabetti, E.; Sandri, M.; Friso, S.; Pizzolo, F.; Schaeffer, L.; Heinrich, J.; et al. FADS genotypes and desaturase activity estimated by the ratio of arachidonic acid to linoleic acid are associated with inflammation and coronary artery disease. Am. J. Clin. Nutr. 2008, 88, 941-949. [CrossRef]

40. Eriksson, L.; Trygg, J.; Wold, S. CV-ANOVA for significance testing of PLS and OPLS ${ }^{\circledR}$ models. J. Chemom. 2008, 22, 594-600. [CrossRef]

41. Efron, B.; Gong, G. A Leisurely Look at the Bootstrap, the Jackknife, and Cross-Validation. Am. Stat. 1983, $37,36-48$.

42. Abraham, T.M.; Pedley, A.; Massaro, J.M.; Hoffmann, U.; Fox, C.S. Association between visceral and subcutaneous adipose depots and incident cardiovascular disease risk factors. Circulation 2015, 132, 1639-1647. [CrossRef] [PubMed] 
43. MacIntyre, U.; Kruger, H.; Venter, C.; Vorster, H. Dietary intakes of an African population in different stages of transition in the North West Province, South Africa: The THUSA study. Nutr. Res. 2002, 22, 239-256. [CrossRef]

44. Garaulet, M.; Pérez-Llamas, F.; Pérez-Ayala, M.; Martínez, P.; Sánchez de Medina, F.; Tebar, F.J.; Zamora, S. Site-specific differences in the fatty acid composition of abdominal adipose tissue in an obese population from a Mediterranean area: Relation with dietary fatty acids, plasma lipid profile, serum insulin, and central obesity. Am. J. Clin. Nutr. 2001, 74, 585-591. [CrossRef]

45. Gower, B.A.; Alvarez, J.A.; Bush, N.C.; Hunter, G.R. Insulin sensitivity affects propensity to obesity in an ethnic-specific manner: Results from two controlled weight loss intervention studies. Nutr. Metab. 2013, 10, 3. [CrossRef]

46. Gianmattia, G.; Ferreri, C.; Marfella, R.; Pournaras, D.; Roux, C.; del Genio, F.; Paolo, L.; Tolone, S.; Docimo, L.; Puca, A.A. Morbid Obesity is Associated to Altered Fatty Acid Profile of Erythrocyte Membranes. J. Diabetes Metab. 2015, 6, 1-4.

47. Cordero, A.; González, J.E.; Perona, J.S.; Padilla, L.; Ferre, A.; Hita, O.; Baeza, R.; Barrilao, G.; Rivas, G. Obesity and its relation with markers of inflammation and erythrocyte fatty acids in a group of overweight adolescents. Nutr. Hosp. 2012, 27, 161-164.

48. Mika, A.; Sledzinski, T. Alterations of specific lipid groups in serum of obese humans: A review. Obes. Rev. 2017, 18, 247-272. [CrossRef]

49. Mika, A.; Kaska, L.; Korczynska, J.; Mirowska, A.; Stepnowski, P.; Proczko, M.; Ratnicki-Sklucki, K.; Goyke, E.; Sledzinski, T. Visceral and subcutaneous adipose tissue stearoyl-CoA desaturase-1 mRNA levels and fatty acid desaturation index positively correlate with BMI in morbidly obese women. Eur. J. Lipid Sci. Technol. 2015, 117, 926-932. [CrossRef]

50. Ojwang, A.A.; Kruger, H.S.; Zec, M.; Ricci, C.; Pieters, M.; Kruger, I.M.; Wentzel-Viljoen, E.; Smuts, C.M. Plasma phospholipid fatty acid patterns are associated with adiposity and the metabolic syndrome in black South Africans: A cross-sectional study. Cardiovasc. J. Afr. 2019, 30, 228-238. [CrossRef]

51. Malcom, G.T.; Bhattacharyya, A.K.; Velez-Duran, M.; Guzman, M.A.; Oalmann, M.C.; Strong, J.P. Fatty acid composition of adipose tissue in humans: Differences between subcutaneous sites. Am. J. Clin. Nutr. 1989, 50, 288-291. [CrossRef] [PubMed]

52. Phinney, S.D.; Stern, J.S.; Burke, K.E.; Tang, A.B.; Miller, G.; Holman, R.T. Human subcutaneous adipose tissue shows site-specific differences in fatty acid composition. Am. J. Clin. Nutr. 1994, 60, 725-729. [CrossRef]

53. Calder, P.C.; Harvey, D.J.; Pond, C.M.; Newsholme, E.A. Site-specific differences in the fatty acid composition of human adipose tissue. Lipids 1992, 27, 716-720. [CrossRef] [PubMed]

54. Wang, L.; Manson, J.E.; Rautiainen, S.; Gaziano, J.M.; Buring, J.E.; Tsai, M.Y.; Sesso, H.D. A prospective study of erythrocyte polyunsaturated fatty acid, weight gain, and risk of becoming overweight or obese in middle-aged and older women. Eur. J. Nutr. 2016, 55, 687-697. [CrossRef] [PubMed]

55. Amri, E.Z.; Ailhaud, G.; Grimaldi, P.A. Fatty acids as signal transducing molecules: Involvement in the differentiation of preadipose to adipose cells. J. Lipid Res. 1994, 35, 930-937. [PubMed]

56. Aoun, M.; Michel, F.; Fouret, G.; Schlernitzauer, A.; Ollendorff, V.; Wrutniak-Cabello, C.; Cristol, J.P.; Carbonneau, M.A.; Coudray, C.; Feillet-Coudray, C. A grape polyphenol extract modulates muscle membrane fatty acid composition and lipid metabolism in high-fat-high-sucrose diet-fed rats. Br. J. Nutr. 2011, 106, 491-501. [CrossRef]

57. Micallef, M.; Munro, I.; Phang, M.; Garg, M. Plasma n-3 Polyunsaturated Fatty Acids are negatively associated with obesity. Br. J. Nutr. 2009, 102, 1370-1374. [CrossRef]

58. Huang, T.; Bhulaidok, S.; Cai, Z.; Xu, T.; Xu, F.; Wahlqvist, M.L.; Li, D. Plasma phospholipids n-3 polyunsaturated fatty acid is associated with metabolic syndrome. Mol. Nutr. Food Res. 2010, 54, 1628-1635. [CrossRef]

59. Fickova, M.; Hubert, P.; Crémel, G.; Leray, C. Dietary (n-3) and (n-6) polyunsaturated fatty acids rapidly modify fatty acid composition and insulin effects in rat adipocytes. J. Nutr. 1998, 128, 512-519. [CrossRef]

60. Aglago, E.K.; Biessy, C.; Torres-Mejia, G.; Angeles-Llerenas, A.; Gunter, M.J.; Romieu, I.; Chajes, V. Association between serum phospholipid fatty acid levels and adiposity in Mexican women. J. Lipid Res. 2017, 58, 1462-1470. [CrossRef] 
61. Mayneris-Perxachs, J.; Guerendiain, M.; Castellote, A.I.; Estruch, R.; Covas, M.I.; Fito, M.; Salas-Salvado, J.; Martinez-Gonzalez, M.A.; Aros, F.; Lamuela-Raventos, R.M. Plasma fatty acid composition, estimated desaturase activities, and their relation with the metabolic syndrome in a population at high risk of cardiovascular disease. Clin. Nutr. 2014, 33, 90-97. [CrossRef] [PubMed]

62. Kabagambe, E.K.; Tsai, M.Y.; Hopkins, P.N.; Ordovas, J.M.; Peacock, J.M.; Borecki, I.B.; Arnett, D.K. Erythrocyte fatty acid composition and the metabolic syndrome: A National Heart, Lung, and Blood Institute GOLDN study. Clin. Chem. 2008, 54, 154-162. [CrossRef] [PubMed]

63. Yary, T.; Voutilainen, S.; Tuomainen, T.P.; Ruusunen, A.; Nurmi, T.; Virtanen, J.K. Omega-6 polyunsaturated fatty acids, serum zinc, delta-5- and delta-6-desaturase activities and incident metabolic syndrome. J. Hum. Nutr. Diet. 2017, 30, 506-514. [CrossRef]

64. Rosqvist, F.; Bjermo, H.; Kullberg, J.; Johansson, L.; Michaelsson, K.; Ahlstrom, H.; Lind, L.; Riserus, U. Fatty acid composition in serum cholesterol esters and phospholipids is linked to visceral and subcutaneous adipose tissue content in elderly individuals: A cross-sectional study. Lipids Health Dis. 2017, 16, 68. [CrossRef] [PubMed]

65. Gertow, K.; Rosell, M.; Sjogren, P.; Eriksson, P.; Vessby, B.; de Faire, U.; Hamsten, A.; Hellenius, M.L.; Fisher, R.M. Fatty acid handling protein expression in adipose tissue, fatty acid composition of adipose tissue and serum, and markers of insulin resistance. Eur. J. Clin. Nutr. 2006, 60, 1406-1413. [CrossRef]

66. Iggman, D.; Arnlov, J.; Vessby, B.; Cederholm, T.; Sjogren, P.; Riserus, U. Adipose tissue fatty acids and insulin sensitivity in elderly men. Diabetologia 2010, 53, 850-857. [CrossRef]

(C) 2020 by the authors. Licensee MDPI, Basel, Switzerland. This article is an open access article distributed under the terms and conditions of the Creative Commons Attribution (CC BY) license (http://creativecommons.org/licenses/by/4.0/). 